\title{
PERFECT PELL POWERS
}

\author{
by J. H. E. COHN
}

(Received 9 May 1994)

In the thirty years since it was proved that 0,1 and 144 were the only perfect squares in the Fibonacci sequence $[1,9]$, several generalisations have been proved, but many problems remain. Thus it has been shown that 0,1 and 8 are the only Fibonacci cubes [6] but there seems to be no method available to prove the conjecture that $0,1,8$ and 144 are the only perfect powers.

In a different direction, generalising the sequence to $P_{n}(a)$ defined by $P_{0}(a)=0$, $P_{1}(a)=1, P_{n+2}(a)=a P_{n+1}(a)+P_{n}(a)$ or to $p_{n}(a)$ defined by $p_{0}(a)=0, p_{1}(a)=1$, $p_{n+2}(a)=a p_{n+1}(a)-p_{n}(a)$, it has been shown that the problem of determining the squares in these sequences can be handled easily when $a$ is odd, but only in exceptional cases when $a$ is even $[2,3,4]$. In the case of the first of these with $a=2$, we obtain the Pell sequence, $0,1,2,5, \ldots, 169, \ldots$, to which we shall refer below simply as $P_{n}$. It has been shown by Ljunggren [5] that its only squares are 0,1 and 169. However, the method of that paper was long and extremely complicated, involving relative units in a biquadratic field, and Mordell asked over 30 years ago [7] whether a simpler proof might not be available. There has indeed been another proof recently [8] which is quite different in conception, depending as it does on purely analytical ideas. Although that proof is a considerable achievement, whether it can be regarded as more simple is a matter of opinion, as it still seems to require tools and a mass of detail disproportionate to the apparent difficulty of the problem. Maybe what Mordell had in mind was a proof akin to that for Fibonacci squares, both short and technically elementary.

Despite this challenge, no such proof has appeared; it may therefore perhaps be of interest to present the following very simple proof of the fact that there are no other powers in the sequence, a result far exceeding the present state of knowledge of the corresponding problem for the Fibonacci sequence.

THEOREM. The only solutions of $P_{n}=x^{k}$ with $k>2$ are given by $n=0,1$.

LEMma. The Diophantine equation $y^{2}-2 z^{k}=-1$ with $k>2$ has only the solutions $y=z=1$ and $y=239, z=13, k=4$.

Proof of lemma. For $k=4$ or a multiple of 4 , the result is Ljunggren's. For other values, $k$ must have an odd prime factor, and so without loss of generality may be taken to be odd, say $k=2 K+1$. For any solution both $y$ and $z$ must be odd, and factorising in $Q[i]$ gives $(y+i)(y-i)=(1+i)(1-i) z^{2 K+1}$. Since $(1+i)$ and $(1-i)$ are associates we find that $y+i=(1+i)(a+i b)^{2 K+1}$ and $z=a^{2}+b^{2}$ for some suitable rational integers $a$ and $b$, since any units, i.e. powers of $i$, can be absorbed into the $a+i b$. Thus we find $2 i=(1+i)(a+i b)^{2 K+1}-(1-i)(a-i b)^{2 K+1}$ and so

$$
\begin{aligned}
1+i & =(a+i b)^{2 K+1}+i(a-i b)^{2 K+1} \\
& =(a+i b)^{2 K+1}+(-1)^{K}(i a+b)^{2 K+1} .
\end{aligned}
$$

Thus, if $K$ is even, $(1+i)$ is divisible by $(a+i b)+(i a+b)=(1+i)(a+b)$ whence

Glasgow Math. J. 38 (1996) 19-20. 
$a+b= \pm 1$, and similarly, if $K$ is odd, $a-b= \pm 1$. In either case we obtain $z=a^{2}+b^{2}=$ $2 a^{2} \pm 2 a+1$, and so $2 z=c^{2}+1$, where $c=|2 a \pm 1| \geq 1$.

Our equation can now be rewritten in the form $y^{2}-\left(c^{2}+1\right)\left(z^{K}\right)^{2}=-1$, and since the general solution of the Pell equation $u^{2}-\left(c^{2}+1\right) v^{2}=-1$ is given by

$$
u+v \sqrt{c^{2}+1}=\left(c+\sqrt{c^{2}+1}\right)^{2 m+1},
$$

we find that

$$
z^{K}=\left(\frac{1}{2}\left(c^{2}+1\right)\right)^{K}=\sum_{r=0}^{m}\left(\begin{array}{c}
2 m+1 \\
2 r+1
\end{array}\right) c^{2 m-2 r}\left(c^{2}+1\right)^{r}
$$

Now suppose that $p$ is any prime dividing $\frac{1}{2}\left(c^{2}+1\right)$. Then $p \geq 5$. Let $p^{\wedge} \| \frac{1}{2}\left(c^{2}+1\right)$. Then from (1) we see that $p \mid(2 m+1)$ and so if $p^{\mu} \|(2 m+1)$, we see that the first term on the right hand side of (1) is divisible by $p^{\mu}$ precisely, whereas all the other terms are divisible by higher powers. Thus $\lambda K=\mu$, and since this holds for every prime factor of $\frac{1}{2}\left(c^{2}+1\right)$, it follows that $\left(\frac{1}{2}\left(c^{2}+1\right)\right)^{K}$ divides $(2 m+1)$ and so $2 m+1 \geq\left(\frac{1}{2}\left(c^{2}+1\right)\right)^{K}$. On the other hand from (1) we see that $\left(\frac{1}{2}\left(c^{2}+1\right)\right)^{K}>2 m+1$ unless $m=0$ and $c=1$. Thus $z=1$.

Proof of theorem. For $n$ odd, the result follows from the lemma and the identity $Q_{n}^{2}-2 P_{n}^{2}=(-1)^{n}$ where the sequence $Q_{n}$ satisfies the same recurrence relation as $P_{n}$ but with initial conditions $Q_{0}=Q_{1}=1$. For $n$ even, $n \neq 0$, let $n=2^{h} m$, where $m$ is odd. Then it is found without difficulty that $h \geqslant 2$ and that $P_{n}=2^{h} P_{m} Q_{m} Q_{2 m} X$, where the five factors on the right are pairwise coprime. It thus follows that if $P_{n}$ is to be a perfect $k$ th power, then each factor on the right must also be one. But by the lemma $P_{m}$ can be a perfect $k$ th power only if $m=1$, and then $Q_{2 m}=3$ fails to be one, which concludes the proof.

\section{REFERENCES}

1. J. H. E. Cohn, On Square Fibonacci Numbers, J. London Math. Soc. 39 (1964) 537-540.

3. J. H. E. Cohn, Five Diophantine Equations, Math. Scand. 21 (1967) 61-70.

4. J. H. E. Cohn, Squares in some recurrent sequences, Pacific J. Math. 41 (1972) 631-646. 5 (1942).

5. W. Ljunggren, Zur Theorie de Gleichung $x^{2}+1=D y^{4}$, Avh. Norske Vid. Akad., Oslo 1, No.

6. Hymie London and Raphael Finkelstein, On Fibonacci and Lucas numbers which are perfect powers, Fibonacci Quart. 5 (1969) 476-481. 161-164.

7. L. J. Mordell, The diophantine equation $y^{2}=D x^{4}+1$, J. London Math. Soc. 39 (1964)

8. Ray Steiner and Nikos Tzanakis, Simplifying the solution of Ljunggren's equation $X^{2}+1=2 Y^{4}$, J. Number Theory, 37 (1991) 123-132.

9. O. Wyler, Solution to Problem 5080, Amer. Math. Monthly 71 (1964) 220-222.

Department of Mathematics,

ROYAL HOLLOWAY UNIVERSITY OF LONDON, Egham, SURREY TW20 0EX

ENGLAND 